\title{
Endothelial Dysfunction for Acute Kidney Injury in Coronavirus Disease 2019: How Concerned Should We Be?
}

\author{
Yung-Chang Chen ${ }^{a, b}$ Ji-Tseng Fang ${ }^{a, b}$ Chih-Wei Yang ${ }^{a, b}$ \\ ${ }^{a}$ Department of Nephrology, Kidney Research Center, Taipei, Taiwan; ${ }^{b}$ Chang Gung University College of Medicine, \\ Taipei, Taiwan
}

\section{Keywords}

Acute kidney injury · Coronavirus disease 2019 - Endothelial dysfunction · Intensive care unit

\begin{abstract}
Context: The outbreak of coronavirus disease 2019 (COVID-19) has rapidly evolved into a global pandemic. Kidney dysfunction is common among patients with COVID-19, and patients who develop acute kidney injury (AKI) have inferior outcomes. There is a growing body of evidence that AKI occurs in a substantial number of patients with COVID-19 and that developing AKI is associated with significantly worse outcomes for COVID-19 patients. The risk for death was amplified when AKI resulted in kidney replacement therapy (KRT). Subject of Review: The Study of the Treatment and Outcomes in Critically III Patients with COVID-19 (STOP-COVID) conducted a multicenter retrospective observational study enrolling 3,099 critically ill adults with COVID-19 admitted to intensive care units (ICUs) (J Am Soc Nephrol 2021;32:161-176). A total of 637 of 3,099 patients (20.6\%) developed AKI treated with KRT (AKI-KRT) within 14 days of ICU admission, 350 of whom (54.9\%) died within 28 days of ICU admission. Predictors of COVID-19 patients' progress to
\end{abstract} of the partial pressure of arterial oxygen over the fraction of inspired oxygen ( $\mathrm{PaO2}: \mathrm{FiO} 2$ ratio) on ICU admission, and greater number of vasopressors received on ICU admission. Second Opinion: Recently, some investigations revealed that the independent predictors of COVID-19 with AKI include older age, Black race, diabetes, hypertension, cardiovascular disease, mechanical ventilation, higher interleukin-6 level, and use of vasopressor medications. It seems that the underlying comorbidities with preexisting vascular endothelial damage and/or the more serious critically ill COVID-19 patients can contribute to the development of AKI and even AKI-KRT.

(c) 2021 S. Karger AG, Basel

\section{Introduction}

Coronavirus disease 2019 (COVID-19) mainly affecting respiratory systems has become a pandemic and spread worldwide. Acute kidney injury (AKI) has been reported as a severe complication of COVID-19 with a very high risk of mortality $[1,2]$. In a meta-analysis of approximately 6,945 mostly hospitalized patients from Chi-

Correspondence to:

Yung-Chang Chen, cyc2356@adm.cgmh.org.tw 
na, Italy, the UK, and the USA, the incidence of AKI was 8.9\% (95\% CI: 4.6-14.5) [3]. Nearly 9 out of 100 developed AKI among those COVID-19 patients. This was close to the incidence rate of AKI in patients with community-acquired pneumonia [4]. Of note, kidney replacement therapy (KRT) was used in near $5 \%$ of the critically ill patients [5]. COVID-19 patients with AKI had around 5.6 times mortality risk than those without AKI [6].

\section{Epidemiology and Risk Factors}

The most common chronic comorbidities in deceased COVID-19 patients were diabetes mellitus (DM) (31.7\%), chronic obstructive pulmonary disease (18.1\%), hypertension (70\%), atrial fibrillation (22.5\%), chronic kidney disease (CKD) (23.1\%), active cancer within the previous 5 years $(16.8 \%)$, ischemic heart disease $(16 \%)$, and obesity (10\%) [7]. CKD was present in $>20 \%$ deceased COVID-19 patients. In addition to the presence of CKD, it also has been verified that COVID-19 patients are at increased risk of developing AKI [7].

AKI during COVID-19 infection is associated with high mortality rates in the intensive care unit (ICU). Data from China and the USA suggest that male sex, older age, Black race, DM, CKD, hypertension, cardiovascular disease, congestive heart failure ( $\mathrm{CHF}$ ), and higher $\mathrm{BMI}$ are associated with AKI in COVID-19 [8]. Among COVID-19 patients, those with AKI are more likely to need vasopressors and mechanical ventilation [8].

Endothelial dysfunction is present in several cardiovascular and metabolic diseases, such as hypertension, $\mathrm{CHF}$, peripheral arterial disease, atherosclerosis, DM, obesity, septic shock, and CKD [9]. Damage to the endothelium in the vessels and/or kidneys may play a key role in the reversibility of ICU COVID-19 patient-related AKI [8].

\section{The Important Role of Endothelial Dysfunction for} AKI in COVID-19

Endothelial dysfunction characterized by high D-dimer levels and microvascular damage represents an important risk factor for COVID-19-related coagulopathy [8]. An intact endothelium produces a variety of vasoactive substances that control enhanced vasoconstriction, modulate platelet-vessel wall interaction, and help in adherence of monocytes and migration as well as proliferation of vascular smooth muscle cells. Strategically located between the circulating blood and the vascular smooth muscle, the renal endothelium also closely interacts with mesangial, tubular, and juxtaglomerular cells and may thus contribute to the regulation of renal vascular tone and renal function [9]. Therefore, the endothelium is not just a permeability barrier and target but is increasingly recognized as a mediator in the pathogenesis of cardiovascular and renal disease [9].

Impairment of endothelial function develops long before structural changes occur and is associated with cardiovascular risk factors such as hypercholesterolemia, hypertension, diabetes, and aging [9]. Besides, an increasing body of evidence indicates that endothelial dysfunction can be added to the list of risk factors that contribute to vascular injury in patients with CKD [9]. Renal endothelial cells contribute to vascular tone, regulation of blood flow to local tissue beds, and modulation of coagulation and inflammation accompanied by vascular permeability. Both ischemia and sepsis have attentive effects on the renal endothelium, resulting in microvascular dysregulation consequentially persistent ischemia and further injury. Therefore, endothelial dysfunction would be associated with higher incidence, worsened severity, and prolonged duration of AKI after COVID-19 infection [8].

\section{Pathophysiology of AKI in COVID-19}

The cause of kidney involvement in COVID-19 is likely to be multifactorial (Table 1) [8]. The pathophysiology of AKI in COVID-19 now relies on the following:

1. Fluid balance disturbances leading to prerenal AKI: prolonged fever, tachypnea, and gastrointestinal problems (vomiting or diarrhea) promoting hypovolemia $[1,10]$.

2. Imbalanced renin-angiotensin-aldosterone system (RAAS) activation: COVID-19 can directly infect the renal tubular epithelium and podocytes through the RAAS pathway. Consequently, COVID-19-mediated angiotensin II accumulation may promote an imbalanced RAAS activation, leading to inflammation, fibrosis, and vasoconstriction $[10,11]$.

3. Toxic tubular damage following cytokine release syndrome or rhabdomyolysis: viral infection in alveolar cells results in massive recruitment of immune cells, which produce large amounts of cytokines that can mediate cytokine-mediated hypoperfusion-related acute renal tubules injury. Cytokine storm might also contribute to the pathogenesis of COVID-19-associated multiorgan dysfunction. Besides, rhabdomyolysis which occurred in COVID-19 patients with skeletal muscle injury has been reported $[7,11]$. 
Table 1. Pathogenesis of AKI in COVID-19

Direct viral effects: COVID-19 might display viral tropism and directly affect the kidney

Collapsing glomerulopathy: direct viral effect or cytokines lead to FSGS, APOL1 alleles are a genetic risk factor Endothelial damage: virus particles were reported to injure renal endothelial cells

Coagulopathy: may be through the hypoxia-inducible transcription factor-dependent signaling pathway

Complement activation: through the lectin pathway has been described

Inflammation: COVID-19-associated multiorgan dysfunction

Indirect effects
Mechanical ventilation: PEEP
Hypovolemia: fever, tachypnea, vomiting, diarrhea, or restrictive fluid strategy
Organ crosstalk: lung-kidney axis and heart-kidney axis or cardiorenal syndrome
Acute tubular injury: hemodynamic instability, rhabdomyolysis, or secondary sepsis inducing cytokine storm
Nephrotoxins: radiographic contrast media, antibiotics, or antiviral therapy

AKI, acute kidney injury; COVID-19, coronavirus disease 2019; FSGS, focal segmental glomerulosclerosis; PEEP, positive end-expiratory pressure.

4. Endotheliitis, thrombotic events, and intravascular coagulation: virus particles were reported to be present in renal endothelial cells, indicating viremia as a possible reason of endothelial damage in the kidney. Apart from this, the mainly severe COVID-19 patients present signs of increased coagulation activity, resulting in consumption of coagulation factors and disseminated microvascular thrombosis. Hypoxia associated with COVID-19 pneumonia increases blood viscosity both directly and through the hypoxia-inducible transcription factor-dependent signaling pathway thereby inducing thrombosis. The high incidence of acute thrombotic events in COVID-19 patients has been reported by several studies, mostly venous thrombosis and pulmonary embolism [8].

5. A complex process driven by virus-mediated injury: complement activation through the lectin pathway has already been described in COVID-19-infected patients. Obviously, complement activation and thrombotic microangiopathy are important mechanisms of AKI in COVID-19 [11].

6. Organ crosstalk: in the lung-kidney axis, several physiological studies suggested that high intrathoracic pressures and positive end-expiratory pressure could influence central venous return, urine output, and glomerular filtration. The heart-kidney axis or cardiorenal syndrome, particularly right ventricular failure secondary to COVID-19 pneumonia, might lead to kidney congestion, and left ventricular dysfunction might induce low cardiac output, arterial underfilling, and kidney hypoperfusion [10].
7. Drug nephrotoxicity: radiographic contrast media used to examine thromboembolic events (such as pulmonary embolism) may play a role in AKI onset. Nephrotoxic drugs can also be implicated in AKI progress, especially antibiotics, antiviral therapy, or traditional medicine $[7,10]$.

\section{Management for COVID-19 Patients with AKI}

COVID-19 is associated with a high incidence of AKI [3]. Management of patients with a confirmed diagnosis of AKI in COVID-19 initiates with an evaluation of the cause of AKI [10]. Supportive treatment includes optimizing hemodynamics, adequate volume status management, and discontinuation of nephrotoxic medications. Timing, modality, and dose of KRT for patients with COVID-19-associated AKI are similar to other critically ill AKI patients [8]. Continuous kidney replacement therapy (CKRT) is the preferred modality of dialysis in patients with hypotension [8]. Hospital dialysis units may experience a lack of dialysis supplies, dialysis technicians, and nurses. In the situation of an acute shortage of other KRT modalities and personnel, using acute peritoneal dialysis may be taken into consideration [12]. Theoretically, convective forms of KRT (hemofiltration) have been suggested to increase the cytokine removal, but they have not been recognized to have better outcomes than diffusive forms (hemodialysis) [12]. Hypercoagulability poses a major problem in patients with COVID-19, and suitable anticoagulation should be considered for all KRT patients [8].

Apart from need for KRT in severe AKI, there are modalities for extracorporeal blood purification in CO- 
Table 2. Extracorporeal blood purification and cytokine removal in COVID-19

\begin{tabular}{ll}
\hline Techniques & Treatment strategies and target molecule removal \\
\hline KRT & $\begin{array}{l}\text { Remove target molecules by adsorption (AN69 or PMMA), remove target molecules by diffusion or convection } \\
\text { (MCO or HCO), electrolyte disorder, metabolic acidosis, fluid overload, and uremic toxins }\end{array}$ \\
$\begin{array}{ll}\text { Hemoperfusion } \\
\text { TPE }\end{array}$ & $\begin{array}{l}\text { Inflammatory molecules, DAMPs, and PAMPs, including COVID-19 particles } \\
\text { Inflammatory mediators and proteins associated with hypercoagulability }\end{array}$ \\
\hline Cartridges & Target molecule removal \\
\hline $\begin{array}{l}\text { CytoSorb } \\
\text { oXiris (AN69) }\end{array}$ & $\begin{array}{l}\text { Cytokines (hemoadsorption) } \\
\text { Polymyxin-B }\end{array}$ \\
ECO & $\begin{array}{l}\text { Endotoxin and cytokines (hemoadsorption) } \\
\text { Range of 20- to 50-kDa inflammatory cytokines (diffusion or convection) }\end{array}$ \\
\hline
\end{tabular}

COVID-19, coronavirus disease 2019; KRT, kidney replacement therapy; PMMA, polymethylmethacrylate; MCO, medium cutoff; HCO, high cutoff; DAMPs, damage-associated molecular patterns; PAMPs, pathogen-associated molecular patterns; TPE, total plasma exchange.

VID-19 patients that are being used for experimental treatment such as cytokine adsorber and plasma exchange or infusion of convalescent plasma (Table 2) $[8,13]$. Hemoperfusion involves nonspecific removal of cytokines by an extracorporeal membrane and has been proposed as a complementary therapeutic option in patients with COVID-19 and multiorgan dysfunction [10]. Four different approaches can be used for cytokine removal: (1) direct hemoperfusion using a neutro-macroporous sorbent; (2) plasma adsorption on a resin after plasma separation from whole blood; (3) CKRT with hollow fiber filters with adsorptive properties; and (4) high-dose CKRT with medium cutoff or high cutoff membranes. All these modalities can be used in conjunction with CKRT to treat the multiorgan failure usually seen in critically ill patients with COVID-19 [13]. However, hemoadsorption devices have been shown to remove cytokines such as interleukin-6, but this may not translate to improve patient outcomes. The extent to which hemoperfusion might be beneficial for COVID-19 remains unclear, and this has not yet been evaluated in a randomized trial [12].

\section{Conclusion}

AKI in COVID-19 is associated with increased mortality. Independent risk factors for AKI were male gender, elderly people, Black race, $\mathrm{DM}, \mathrm{CKD}$, hypertension, cardiovascular disease, CHF, and higher BMI. Preexisting endothelial dysfunction may play a main role for AKI in COVID-19. The pathophysiology is multifactorial, and management is supportive, with possible need for extracorporeal therapies for critically ill patients. Large-scale prospective clinical trials can provide optimal management of AKI in COVID-19, as well as more retrospective data on clinical experience are needed to judge the impact.

\section{Conflict of Interest Statement}

The authors declare that they have no conflicts of interest to disclose.

\section{Author Contributions}

Y.C. Chen: writing; J.T. Fang: discussion of opinions; C.W. Yang: providing comments and proofreading.

References

1 Naicker S, Yang CW, Hwang SJ, Liu BC, Chen JH, Jha V. The novel coronavirus 2019 epidemic and kidneys. Kidney Int. 2020;97(5): 824-8.

2 Chen YT, Shao SC, Lai EC, Hung MJ, Chen YC. Mortality rate of acute kidney injury in SARS, MERS, and COVID-19 infection: a systematic review and meta-analysis. Crit Care. 2020;24(1):439.

3 Chen YT, Shao SC, Hsu CK, Wu IW, Hung $\mathrm{MJ}$, Chen YC. Incidence of acute kidney injury in COVID-19 infection: a systematic review and meta-analysis. Crit Care. 2020; 24(1):346. 
4 Akram AR, Singanayagam A, Choudhury G, Mandal P, Chalmers JD, Hill AT. Incidence and prognostic implications of acute kidney injury on admission in patients with community-acquired pneumonia. Chest. 2010; 138(4):825-32.

5 Fisher M, Neugarten J, Bellin E, Yunes M, Stahl L, Johns TS, et al. AKI in hospitalized patients with and without COVID-19: a comparison study. J Am Soc Nephrol. 2020;31(9): 2145-57.

$6 \mathrm{Ng} \mathrm{JH}$, Hirsch JS, Hazzan A, Wanchoo R, Shah HH, Malieckal DA, et al. Northwell nephrology COVID-19 research consortium: outcomes among patients hospitalized with COVID-19 and acute kidney injury. Am J Kidney Dis. 2021;77:204-15.
7 Gagliardi I, Patella G, Michael A, Serra R, Provenzano M, Andreucci M. COVID-19 and the kidney: from epidemiology to clinical practice. J Clin Med. 2020;9(8):2506.

8 Nadim MK, Forni LG, Mehta RL, Connor MJ Jr, Liu KD, Ostermann M, et al. COVID19-associated acute kidney injury: consensus report of the 25th acute disease quality initiative (ADQI) workgroup. Nat Rev Nephrol. 2020;16(12):747-64.

9 Ruschitzka F, Shaw S, Gygi D, Noll G, Barton M, Lüscher TF. Endothelial dysfunction in acute renal failure: role of circulating and tissue endothelin-1. J Am Soc Nephrol. 1999; 10(5):953-62.
10 Ronco C, Reis T, Husain-Syed F. Management of acute kidney injury in patients with COVID-19. Lancet Respir Med. 2020;8(7): 738-42.

11 Izzedine $\mathrm{H}$, Jhaveri KD. Acute kidney injury in patients with COVID-19: an update on the pathophysiology. Nephrol Dial Transplant. 2021;36(2):224-6.

12 Clark EG, Hiremath S, Soroka SD, Wald R, Weir MA. CSN COVID-19 rapid review program: management of acute kidney injury. Can J Kidney Health Dis. 2020; 7: 2054358120941679.

13 Ronco C, Reis T. Kidney involvement in COVID-19 and rationale for extracorporeal therapies. Nat Rev Nephrol. 2020;16(6):308-10. 